Cite this: Phys. Chem. Chem. Phys., 2012, 14, 6487-6495

\title{
Catalytic activity of nanoalloys from gold and palladium $\dagger$
}

\author{
Julian Kaiser, ${ }^{a}$ Linn Leppert, ${ }^{b}$ Hannes Welz, ${ }^{a}$ Frank Polzer, ${ }^{a}$ Stefanie Wunder, ${ }^{a}$ \\ Nelia Wanderka, ${ }^{c}$ Martin Albrecht, ${ }^{d}$ Thomas Lunkenbein, ${ }^{e}$ Josef Breu, ${ }^{e}$ \\ Stephan Kümmel, ${ }^{* b}$ Yan $\mathrm{Lu}^{* a}$ and Matthias Ballauff $* a$
}

Received 12th December 2011, Accepted 7th March 2012

DOI: $10.1039 / \mathrm{c} 2 \mathrm{cp} 23974 \mathrm{~d}$

We present a quantitative study of the catalytic activity of well-defined faceted gold-palladium nanoalloys which are immobilized on cationic spherical polyelectrolyte brushes. The spherical polyelectrolyte brush particles used as carriers for the nanoalloys consist of a solid polystyrene core onto which cationic polyelectrolyte chains of 2-aminoethyl methacrylate are attached.

$\mathrm{Au} / \mathrm{Pd}$ nanoalloy particles with sizes in the range from 1 to $3 \mathrm{~nm}$ have been generated which are homogeneously distributed on the surface of the spherical polyelectrolyte brushes. The reduction of 4-nitrophenol has been chosen as a well-controlled model reaction allowing us to determine the catalytic activity of the nanoalloys as a function of the $\mathrm{Au} / \mathrm{Pd}$ composition. The adsorption behavior was studied by Langmuir-Hinshelwood kinetics. We find a pronounced maximum of the catalytic activity at 75 molar \% Au. A comparison of gold, platinum, palladium and gold-palladium alloy nanoparticles is made in terms of Langmuir-Hinshelwood kinetics. Density functional calculations for $\mathrm{Au} / \mathrm{Pd}$ clusters with up to 38 atoms show that the density of states at the Fermi level increases with increasing Pd content, and that the highest occupied orbitals are associated with Pd atoms. The calculations confirm that small changes in the atomic arrangement can lead to pronounced changes in the particles' electronic properties, indicating that the known importance of surface effects is further enhanced in nanoalloys.

\section{Introduction}

Nanoparticles have received much attention since they constitute a new class of efficient catalysts. ${ }^{1,2}$ Thus, Au-nanoparticles turned out to be highly active in the catalysis of e.g. oxidation reactions despite the fact that the bulk metal is inactive in catalysis. ${ }^{3}$ Recently, the focus has been shifting towards nanoalloys consisting of two noble metals. ${ }^{4-6} \mathrm{Up}$ to now, there has been clear evidence that nanoalloys are in general better catalysts than the neat metals. ${ }^{4}$ However, the reasons

${ }^{a}$ F-I2 Soft Matter and Functional Materials, Helmholtz-Zentrum Berlin für Materialien und Energie, Hahn-Meitner-Platz 1, 14109 Berlin, Germany.E-mail: Matthias.Ballauff@helmholtz-berlin.de, yan.lu@helmholtz-berlin.de; Fax: +49 (0) 30 8062-43071,

Tel: +49 (0) $308062-42308$

${ }^{b}$ Theoretische Physik IV, University of Bayreuth, 95440 Bayreuth, Germany

${ }^{c}$ F-I1 Institute of Applied Materials, Helmholtz-Zentrum Berlin für Materialien und Energie, Hahn-Meitner-Platz 1, 14109 Berlin, Germany

${ }^{d}$ Leibniz Institute for Crystal Growth Berlin, Max-Born-Straße, 12489 Berlin, Germany

${ }^{e}$ Inorganic Chemistry I, University of Bayreuth, 95440 Bayreuth, Germany

$\dagger$ Electronic supplementary information (ESI) available: EDX spectra, PXRD patterns and mean size evaluation of $\mathrm{Au} / \mathrm{Pd}$ particles. Particle mean size vs. molar Au ratio and Langmuir-Hinshelwood kinetics for Pd nanoparticles. See DOI: 10.1039/c2cp23974d for this apparent synergy are not yet fully understood. Evidently, a quantitative analysis of the catalytic activity of nanoalloys presents a considerable experimental challenge: well-defined nanoalloys of comparable size must be prepared and immobilized on a stable support. Moreover, a well-controlled chemical reaction which is easy to follow must be employed to determine the kinetic constants with maximum precision. The mechanism of this reaction must be clear and all kinetic data need to be explained in terms of this mechanism.

Recently, we demonstrated that well-defined nanoalloys can be prepared on the surface of spherical polyelectrolyte brushes (SPB). ${ }^{7,8}$ Fig. 1 displays the main features of the carrier system: the SPB consist of solid colloidal spheres onto which long chains of polyelectrolytes are grafted. ${ }^{9}$ The dense layer of polyelectrolyte chains immobilizes the ions of noble metals which may be reduced under suitable conditions to yield metal nanoparticles or nanoalloys if a mixture of the respective noble metal has been immobilized. The nanoparticles that have a size of $c a$. 1-2 nm are firmly bound by the polyelectrolyte chains and the entire hybrids have been shown to be stable and robust catalysts. ${ }^{8,10-14}$ In particular, the catalytic reactions can be run in an aqueous phase under very mild conditions.

Here we demonstrate that $\mathrm{Au} / \mathrm{Pd}$ nanoalloys can be prepared on SPB and used for a comprehensive test of their catalytic activity. As a model reaction we use the reduction of 


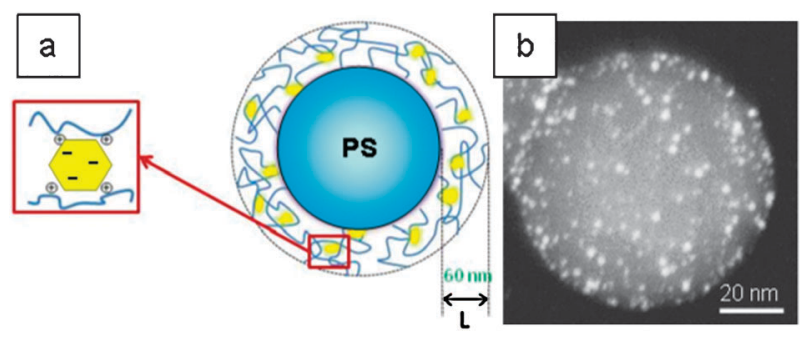

Fig. 1 Schematic presentation of bimetallic Au/Pd NPs immobilised in the spherical polyelectrolyte brushes (SPB). Linear cationic polyelectrolytes of poly-(2-aminoethyl methacrylate) (PAEMH) are densely grafted onto a solid polystyrene (PS) core. (b) Representative STEM image for the Au/Pd@SPB composite particles.

4-nitrophenol (Nip) by borohydride in aqueous solution. ${ }^{13,15-17}$ This reaction has been used intensively for the study of the catalytic activity of nanoparticles in aqueous suspensions. ${ }^{8,10-14,18}$ Moreover, its mechanism has recently been elucidated: ${ }^{13,18}$ it has been demonstrated that the reaction proceeds along a Langmuir-Hinshelwood model, that is, both reactants are adsorbed before they react. Hence, the apparent rate constant derived from the kinetic analysis contains not only the rate constant $k$ for the reaction of the adsorbed species but also the thermodynamic adsorption constants $K_{\mathrm{Nip}}$ and $K_{\mathrm{BH}_{4}}$ for the respective educts. A further point commanding attention was the long induction period $t_{0}$. The analysis of $t_{0}$ in ref. 18 strongly suggested that this time is needed for a restructuring of the surface of the nanoparticles. In this way the reduction of nitrophenol presents a well-studied model reaction which is ideally suited to determine the catalytic activity of metal nanoparticles. Here we apply this reaction for the study of the catalytic activity of $\mathrm{Au} / \mathrm{Pd}$ nanoalloys with high precision. The results obtained therefrom can be compared with the data derived from neat metal nanoparticles.

The paper is organized as follows: we first describe the synthesis and the characterization of these particles. Special emphasis is laid on the characterization by high-resolution electron microscopy. ${ }^{19}$ Then we shall present the results of the study of the catalytic activity of these particles using the reduction of nitrophenol as a model reaction. Finally, we present results of a density functional theory based study of $\mathrm{Au} / \mathrm{Pd}$-clusters and discuss electronic properties that are related to catalytic activity.

\section{Experimental}

\section{Materials}

Cetyltrimethylammonium bromide (CTAB, Fluka), 2-aminoethylmethacrylate hydrochloride (AEMH, Polyscience), 2,2'azobis(2-amidinopropane)dihydrochloride (V50, Aldrich), tetrachloroauric acid trihydrate $\left(\mathrm{HAuCl}_{4} 3 \mathrm{H}_{2} \mathrm{O}\right.$, Aldrich), sodium tetrachloropalladate $\left(\mathrm{Na}_{2} \mathrm{PdCl}_{4}\right.$, Aldrich), sodium borohydride $\left(\mathrm{NaBH}_{4}\right.$, Aldrich) and 4-nitrophenol (Nip, Aldrich) were used as received. Styrene (BASF) was purified by $\mathrm{Al}_{2} \mathrm{O}_{3}$ column and stored in a refrigerator. 2-[p-(2-Hydroxy2-methylpropiophenone)]-ethyleneglycol methacrylate (HMEM) was used as the photo-initiator. The synthesis of this compound has been described previously., 90

\section{Methods}

Photo-emulsion polymerization was done in a UV-reactor (Heraeus TQ $150 \mathrm{Z3}$, range of wavelengths 200-600 $\mathrm{nm}$ ). The amount of metal immobilized on the SPB was determined by TGA using a Mettler Toledo STARe system. The samples were first dried under vacuum at $50{ }^{\circ} \mathrm{C}$. Then $\mathrm{ca} .8 \mathrm{mg}$ of the solid composite particles was heated to $800{ }^{\circ} \mathrm{C}$ under a $60 \mathrm{~mL} \mathrm{~min}{ }^{-1}$ nitrogen flow with a heating rate of $10{ }^{\circ} \mathrm{C} \mathrm{min}{ }^{-1}$ and holding temperature at $800{ }^{\circ} \mathrm{C}$ for about 30 minutes. Scanning transmission electron microscopy (STEM) and energy dispersive X-ray analysis (EDX) were done at $200 \mathrm{kV}$ using a Zeiss Libra $200 \mathrm{FE}(200 \mathrm{kV})$ and a CM20 FEG equipped with a Noran Vantage system (Ge-detector) with about $2 \mathrm{~nm}$ beam diameter attached to the STEM equipment. High resolution-TEM (HR-TEM) was made at an objectivelens aberration corrected FEI Titan 80-300 operated at $300 \mathrm{keV}$. The corrector has been tuned to small negative values of the spherical aberration below $-200 \mathrm{~nm}$. In this case atoms are revealed as white dots if the focus is set to small positive values, while they are revealed as dark dots, i.e. in amplitude contrast, at Gaussian focus. The TEM samples were prepared as follows: a drop of liquid was placed on a grid. After drying in air for three minutes the rest of liquid was taken off by a filter paper. X-Ray diffraction (XRD) measurement was performed at $25{ }^{\circ} \mathrm{C}$ on a Panalytical XPERT-PRO diffractometer in reflection mode using $\mathrm{Cu} \mathrm{K} \alpha(\lambda=1.5418 \AA)$ radiation. Dynamic light scattering (DLS) was done using a Peters ALV 4000 light scattering goniometer at a scattering angle of $90^{\circ}$ at room temperature.

\section{Preparation of Au/Pd $@$ SPB nanocomposite particles}

The SPB carrying chains of poly(2-aminoethylmethacrylate hydrochloride) (PAEMH) were synthesized as described previously. ${ }^{20}$ The $\mathrm{Au} / \mathrm{Pd}$ bimetallic nanoparticles were synthesized as follows: in a typical run, $5 \mathrm{~mL}$ of aqueous solutions of $\mathrm{HAuCl}_{4}$ and $\mathrm{Na}_{2} \mathrm{PdCl}_{4}$ were added drop wise to $100 \mathrm{~mL}$ of SPB latex solution (solid content: $0.1 \mathrm{wt} \%$ ) one by one. The total amount of metal salts is $4.5 \times 10^{-5} \mathrm{~mol}$. Afterwards the mixture was stirred for 30 minutes under $\mathrm{N}_{2}$ to remove the oxygen from the liquid and then the metal ions were reduced by a threefold excess of $\mathrm{NaBH}_{4}$. The reduction of the $\mathrm{AuCl}_{4}{ }^{-}$ and $\mathrm{PdCl}_{4}{ }^{2-}$ ions could be followed easily by a change of colour that the turbid latex solution turned from pale yellow to dark red-brown, depending on the composition. After completion of the reaction the suspension was placed in an ultrafiltration cell and a five-fold excess of deionized water was passed through the cell.

\section{Catalytic reduction of 4-nitrophenol using Au/Pd@SPB composite particles as catalyst}

The catalytic runs were performed as described recently ${ }^{13,18}$ by detecting the extinction of 4-nitrophenol via UV-vis spectroscopy using a Lambda 650 spectrometer (Perkin Elmer). For the calculation of the rate constants $k_{\text {app }}$ of the catalytic reaction normalized to the surface area in unit volume of the system, the specific surface area of the metal nanoparticles was calculated by statistical analysis of TEM-micrographs, assuming spherical particles. The density of the $\mathrm{Au}-\mathrm{Pd}$ nanoparticle was 
calculated from the equation $\rho_{\mathrm{Au} / \mathrm{Pd}}=\left(m_{\mathrm{Au}}+m_{\mathrm{Pd}}\right) /\left(m_{\mathrm{Au}} / \rho_{\mathrm{Au}}+\right.$ $\left.m_{\mathrm{Pd}} / \rho_{\mathrm{Pd}}\right)$, where $\rho_{\mathrm{Pd}}=12.02 \mathrm{~g} \mathrm{~cm}^{-3}, \rho_{\mathrm{Au}}=19.32 \mathrm{~g} \mathrm{~cm}^{-3}, m_{\mathrm{Au}}$ and $m_{\mathrm{Pd}}$ are the weight percentage of pure $\mathrm{Au}$ and $\mathrm{Pd}$ in the alloy, respectively. We assume that volumes are additive like weights hence the approximation.

\section{Results and discussion}

\section{Preparation and characterization}

The preparation of $\mathrm{Au} / \mathrm{Pd}$ nanoalloys in the presence of SPB particles has been performed in two steps: ${ }^{7,8}$ first, $\mathrm{AuCl}_{4}{ }^{-}$and $\mathrm{PdCl}_{4}{ }^{2-}$ ions are immobilized as counterions within the surface layer of cationic polyelectrolyte chains. Thereafter, reduction by $\mathrm{NaBH}_{4}$ leads to nanoparticles of the $\mathrm{Au} / \mathrm{Pd}$ alloy of a given composition. The composition of the resulting nanoalloy can be adjusted by the ratio of the metal ions in the brush layer. ${ }^{7,8}$ The negative charge of the alloy nanoparticles works as a crosslinker for the polyelectrolyte chains by ionic interaction (see Fig. 1; ref. 7). A representative STEM image of a nanoalloy sample shown in Fig. 1 confirms the generation of bimetallic nanoparticles in SPBs. The nanoparticles which are seen as bright dots in the image are confined on the surface of SPB particles and no coagulation takes place during the synthesis of the nanoparticles on SPBs. All composite particle systems remained stable during the reaction and the subsequent cleaning by ultrafiltration. Detailed information about the Au/Pd@SPB nanocomposites is summarized in Table 1.

Energy dispersive X-ray spectra (EDX) show signals of both $\mathrm{Au}$ and $\mathrm{Pd}$, which confirms that $\mathrm{Au} / \mathrm{Pd}$ nanoparticles generated in SPBs consist of an alloy (shown in Fig. S1 in ESI $\dagger$ ). In addition, powder X-ray diffraction (PXRD) patterns for the $\mathrm{Au} / \mathrm{Pd}$ nanoparticles (shown in Fig. S2 in ESI $\dagger$ ) exhibit a marked broadening of the peaks due to the small size of $\mathrm{Au} / \mathrm{Pd}$ nanoparticles. No obvious separate peak corresponding to Au and Pd crystals has been observed from PXRD measurements. High-resolution transmission electron microscopy (HR-TEM) measurements have been taken for the Au/Pd@SPB composite particles. Fig. 2 displays the HR-TEM images of $\mathrm{Au} / \mathrm{Pd}$ nanoparticles with different compositions, respectively. No indication of a core-shell structure for the $\mathrm{Au} / \mathrm{Pd}$ nanoparticles can be found in the HR-TEM images. ${ }^{21}$ From Fig. 2 the crystal lattice can be clearly observed through the entire particle for all $\mathrm{Au} / \mathrm{Pd}$ systems and $\mathrm{Au} / \mathrm{Pd}$ nanoalloys present a face-centered cubic (fcc) crystalline structure. The nanocrystals are faceted and bound mainly by [111] and [001] facets.

Table 1 Composition of the Au/Pd@SPB nanocomposites

\begin{tabular}{|c|c|c|c|c|}
\hline Sample & $\mathrm{Au} / \mathrm{Pd}_{\text {theor }}{ }^{a}$ & $\mathrm{Au} / \mathrm{Pd}_{\exp }{ }^{b}$ & Metal content $\mathrm{t}^{c} / \mathrm{wt} \%$ & $d_{\mathrm{Au}-\mathrm{Pd}}{ }^{d} / \mathrm{nm}$ \\
\hline $\mathrm{Au}_{25} \mathrm{Pd}_{75}$ & $25 / 75$ & $22 / 78$ & 4.94 & $1.7 \pm 0.2$ \\
\hline $\mathrm{Au}_{50} \mathrm{Pd}_{50}$ & $50 / 50$ & $54 / 46$ & 5.71 & $2.2 \pm 0.2$ \\
\hline $\mathrm{Au}_{75} \mathrm{Pd}_{25}$ & $75 / 25$ & $73 / 27$ & 7.93 & $2.5 \pm 0.2$ \\
\hline
\end{tabular}

${ }^{a}$ Theoretical molar ratio of Au and Pd on the SPB. ${ }^{b}$ Measured molar ratio of $\mathrm{Au} / \mathrm{Pd}$ in bimetallic nanoparticles by EDX. ${ }^{c}$ Total metal content in the nanocomposites measured by Thermogravimetric Analysis (TGA). ${ }^{d}$ Diameter of generated $\mathrm{Au}-\mathrm{Pd}$ bimetallic nanoparticles measured from TEM images.
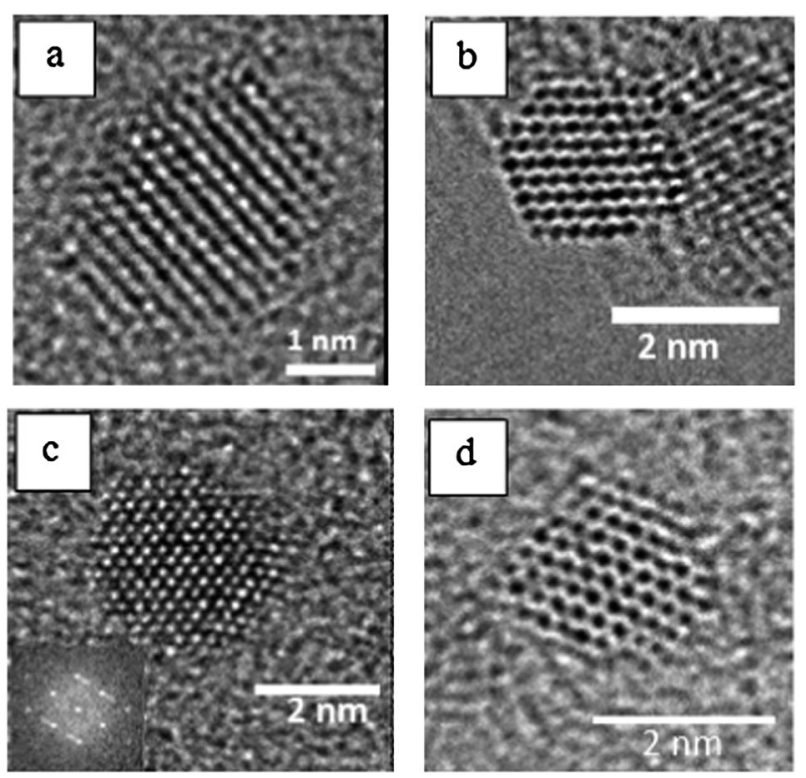

Fig. 2 The representative HR-TEM images of $\mathrm{SPB}-\mathrm{Au}_{25} \mathrm{Pd}_{75}$ (a), $\mathrm{Au}_{50} \mathrm{Pd}_{50}$ (b), $\mathrm{Au}_{75} \mathrm{Pd}_{25}$ (and FFT image of the HR-TEM) (c) and $\mathrm{Au}_{100}$ (d).

The mean size of $\mathrm{Au} / \mathrm{Pd}$ nanoparticles generated in SPBs was evaluated from TEM images (see Fig. S3 in the ESI $\dagger$ ). In all cases, $\mathrm{Au} / \mathrm{Pd}$ alloy particles with a relatively narrow size distribution have been obtained. The average size of the SPB stabilized monometallic $\mathrm{Au}$ and $\mathrm{Pd}$ nanoparticles was found to be $2.8 \pm 0.2 \mathrm{~nm}$ and $1.1 \pm 0.2 \mathrm{~nm}$, respectively, while the $\mathrm{Au} / \mathrm{Pd}$ alloys exhibit an increasing mean particle size with the $\mathrm{Au}$ atomic ratio (see Fig. S4 in ESI $\dagger$ ). A similar tendency has been found for the carbon supported $\mathrm{Au} / \mathrm{Pd}$ nanoalloys as well. ${ }^{22}$

\section{Catalytic activity}

The catalytic reduction of 4-nitrophenol in the presence of an excess of $\mathrm{NaBH}_{4}$ has been chosen as a model reaction. ${ }^{13}$ The kinetics of this reaction can be easily monitored by UV-vis spectroscopy. As shown in Fig. 3, there is an induction time $t_{0}$

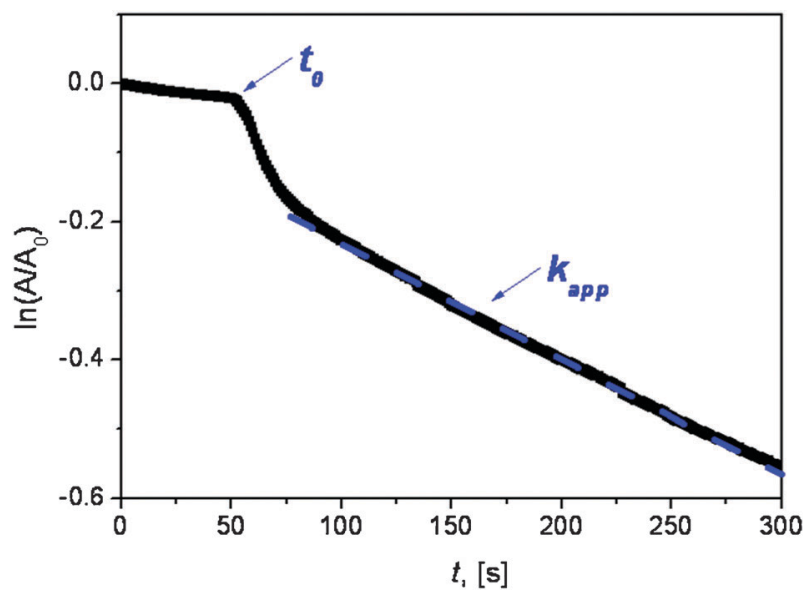

Fig. 3 Time dependence of the absorption of 4-nitrophenolate ions at $400 \mathrm{~nm}$ shown for a typical run. The induction time $t_{0}$ is marked by an arrow. The blue section of the line displays the linear section from which $k_{\text {app }}$ was taken. 


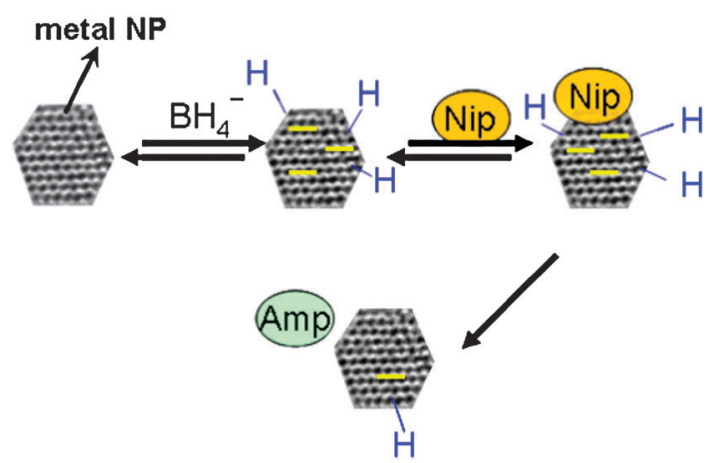

Fig. 4 Scheme of the mechanism of the reduction of 4-nitrophenol (Nip) by borohydride $\left(\mathrm{BH}_{4}^{-}\right)$on the surface of $\mathrm{Au} / \mathrm{Pd}$ nanoalloy crystallite. Both reactants are adsorbed onto the surface and the ratedetermining step is the reaction of the surface hydrogen species with the adsorbed nitrophenol.

in which no reduction takes place. ${ }^{13}$ Then the reaction becomes stationary and the apparent rate constant $k_{\text {app }}$ of the reaction can be determined very precisely from this stationary part by the temporal decay of the strong adsorption of 4-nitrophenol at $400 \mathrm{~nm}$ through

$$
\frac{\mathrm{d} c_{\mathrm{Nip}}}{\mathrm{d} t}=-k_{\mathrm{app}} c_{\mathrm{Nip}}=-k_{1} S c_{\mathrm{Nip}}
$$

where $c_{\mathrm{Nip}}$ is the concentration of 4-nitrophenol, $k_{1}$ is the normalized rate constant, which is the apparent rate $k_{\text {app }}$ normalized to $S$, the total surface area of metal nanoparticles normalized to the unit volume of the solution. As shown schematically in Fig. 4, this reaction takes place on the surface of the particles where both species, namely 4-nitrophenol and surface-hydrogen species generated by borohydride ions react (Langmuir-Hinshelwood kinetics). ${ }^{13,17}$ The surface concentrations of both species are modelled in terms of a Langmuir isotherm. The rate-determining step is the reaction of the surface-bound species while the adsorption/desorption equilibration is assumed to be fast. ${ }^{13,23}$

Given these assumptions, the rate constant $k_{\text {app }}$ is given as ${ }^{13,18}$

$$
k_{\text {app }}=\frac{k S K_{\mathrm{Nip}}^{n} c_{\mathrm{Nip}}^{n-1}\left(K_{\mathrm{BH}_{4}^{-}} c_{\mathrm{BH}_{4}^{-}}\right)^{m}}{\left(1+\left(K_{\mathrm{Nip}} c_{\mathrm{Nip}}\right)^{n}+\left(K_{\mathrm{BH}_{4}^{-}} c_{\mathrm{BH}_{4}^{-}}\right)^{m}\right)^{2}}
$$

where $k$ is the molar rate constant per square metre of the catalyst, which describes the surface reactivity of the adsorbed species, $S$ is the total surface area of metal nanoparticles normalized to the unit volume of the solution, $K_{\mathrm{Nip}}$ is the adsorption constant of 4-nitrophenol, $K_{\mathrm{BH}_{4}}$ is the adsorption constant of borohydride, $c_{\mathrm{Nip}}$ and $c_{\mathrm{BH}_{4}}$ are the concentration of 4-nitrophenol and borohydride, respectively. The exponents $n$ and $m$ are related to the heterogeneity of the sorbents. ${ }^{13}$ Previous results have shown that the adsorption of Nip is modelled best assuming an exponent $n=0.6$ whereas the adsorption of borohydride can be described through the classical Langmuir-isotherm with $m=1 .{ }^{13}$

In the present study, this full kinetic analysis has been performed for the $\mathrm{Au}_{75} \mathrm{Pd}_{25}$ nanoalloy particles by running two sets of experiments as shown in Fig. 5. First, the 4-nitrophenol concentrations are varied at a constant concentration of borohydride $\left(\mathrm{BH}_{4}^{-}\right)(10$ and $5 \mathrm{mM})$. In a second set of kinetic runs
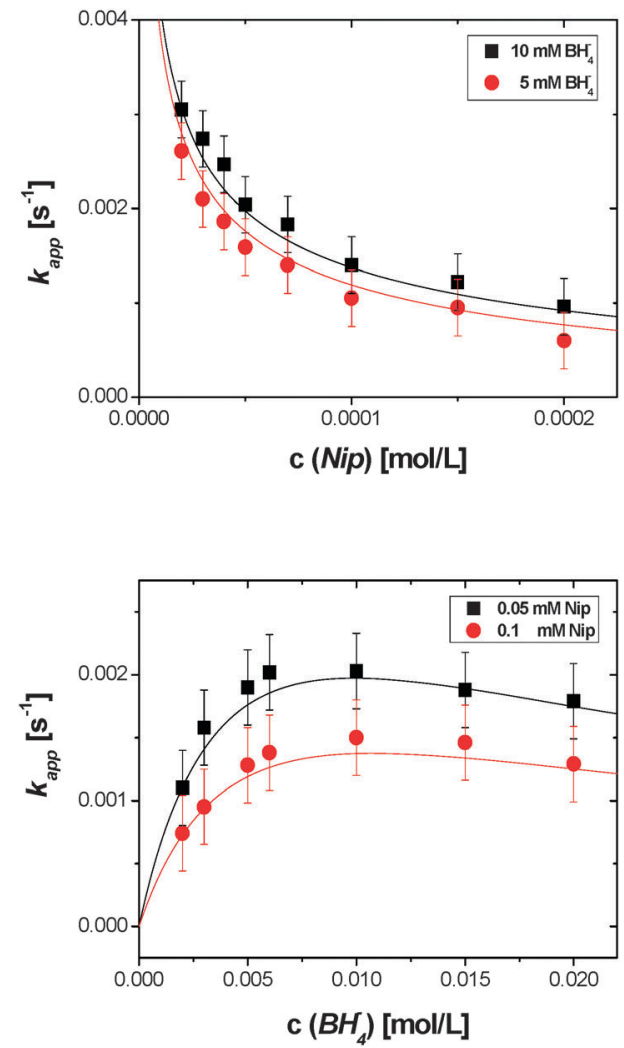

Fig. 5 Dependence of the apparent rate constant $k_{\text {app }}$ on the concentration of Nip (upper panel) and $\mathrm{BH}_{4}{ }^{-}$(lower panel), respectively. The solid lines show the fits of the kinetic model.

the concentrations of $\mathrm{BH}_{4}{ }^{-}$are varied while the concentration of Nip is kept constant $(0.1$ and $0.05 \mathrm{mM})$. The characteristic dependence of $k_{\text {app }}$ on the concentrations of both reactants can be clearly observed for the $\mathrm{Au}_{75} \mathrm{Pd}_{25}$ nanoalloy sample. It reaches a plateau when the concentrations of 4-nitrophenol and borohydride are higher than $0.075 \mathrm{mM}$ and $7.5 \mathrm{mM}$, respectively. The parameters have to be identical for both datasets. The fitting curves react sensitive to changes of $k, K_{\mathrm{Nip}}$ or $K_{\mathrm{BH}_{4}}$. Hence, it is possible to evaluate precise values with narrow uncertainty. The fitting parameters are summarized in Table 2 . These data can be compared directly to previous data obtained by the same analysis for gold $^{18}$ and platinum ${ }^{13}$ nanoparticles: as shown in Table 2, the nanoalloy has the highest kinetic constant $k$, which provides a measure of the rate-determining step. Additionally, palladium nanoparticles were also studied by Langmuir-Hinshelwood kinetics and compared in Table 2. Plots for the dependence of the apparent

Table 2 Comparison of the parameters evaluated by LangmuirHinshelwood kinetics

\begin{tabular}{lllll}
\hline Metal & $k / \mathrm{mol} \mathrm{m}^{-2} \mathrm{~s}^{-1}$ & $K_{\mathrm{Nip}} / \mathrm{L} \mathrm{mol}^{-1}$ & $K_{\mathrm{BH}_{4}} / \mathrm{L} \mathrm{mol}^{-1}$ & $t_{0, \mathrm{sp}} / \mathrm{s}$ \\
\hline $\mathrm{Au}_{75} \mathrm{Pd}_{25}$ & $(2.9 \pm 0.3) \times 10^{-3}$ & $1450 \pm 300$ & $122 \pm 15$ & 685 \\
$\mathrm{Pt}^{13}$ & $(4.6 \pm 0.6) \times 10^{-4}$ & $2300 \pm 500$ & $98 \pm 10$ & 400 \\
$\mathrm{Au}^{18}$ & $(1.6 \pm 0.6) \times 10^{-4}$ & $5500 \pm 1000$ & $58 \pm 5$ & 175 \\
$\mathrm{Pd}$ & $(5.5 \pm 0.5) \times 10^{-5}$ & $2300 \pm 400$ & $48 \pm 5$ & 741
\end{tabular}

$k$ : molar rate constant. $K_{\mathrm{Nip}}$ : adsorption constant of 4-nitrophenol. $K_{\mathrm{BH}_{4}}$ : adsorption constant of borohydride. $t_{\mathrm{o} \text {,sp }}$ : spontaneous induction period. 


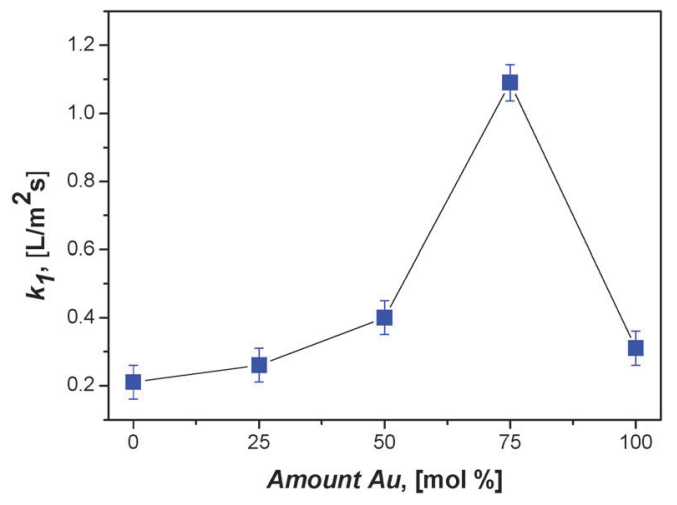

Fig. 6 Catalytic efficiency $k_{1}$ (the normalized rate constant, which is the apparent rate $k_{\text {app }}$ normalized to $S$ as shown in eqn (1)) as the function of the amount of gold in the nanoalloys.

rate constant $k_{\text {app }}$ on the concentration of $\mathrm{Nip}$ and $\mathrm{BH}_{4}{ }^{-}$are shown in Fig. S5 (ESI $\dagger$ ) respectively.

The above results shown in Fig. 5 demonstrate that the apparent constant $k_{\text {app }}$ does not depend strongly on the concentrations of the reactants if a concentration of $0.1 \mathrm{mM}$ of nitrophenol and $10 \mathrm{mM}$ of sodium borohydride is chosen. This is in good agreement with recent findings. ${ }^{13,18}$ Hence, it suffices to perform runs at these concentrations for all nanoalloys under consideration here in order to compare their catalytic activity. For comparison of the different samples, the apparent rates $k_{\text {app }}$ have been normalized to the surface area of the nanoparticles per unit volume. ${ }^{11,24}$ Fig. 6 shows these data as a function of the $\mathrm{Au}$ amount in the system. It demonstrates that nanoalloys have in general higher catalytic activity than the pure Pd-nanoparticles.

\section{Induction period}

An induction period $t_{0}$ has been also observed in present work for gold-palladium nanoalloys and palladium nanoparticles. Previous work on gold and platinum nanoparticles shows similar results which have been explained in terms of surface restructuring. ${ }^{13,18}$ In particular, $1 / t_{0}$ was treated as the rate of this process and analysed using the kinetic data of the stationary reaction..$^{13,18,25}$ Two main results have been obtained from this analysis: (i) $1 / t_{0}$ can be split into a spontaneous part $1 / t_{0, \mathrm{sp}}$ and a part depending on the concentration of nitrophenol; and (ii) the latter part, namely the substrateinduced surface restructuring scales linearly with the square of the degree of surface coverage $\theta_{\mathrm{Nip}}$. The explanation of these findings is obvious: the pristine surface of the nanoparticles is not catalytically active but needs a certain restructuring. ${ }^{18}$ Both the spontaneous as well as the substrate-induced surface restructuring are well-established concepts for planar metallic surfaces $^{26}$ and the precise data obtained from the present model reaction allow us to draw similar conclusions for nanoparticles. ${ }^{18}$

The analysis done here demonstrates that the $\mathrm{Au} / \mathrm{Pd}$ nanoalloys behave in the same way: by plotting the inverse induction time as a function of the concentration of 4-nitrophenol, an extrapolation is made which leads to an intercept $\left(1 / t_{0, \mathrm{sp}}\right)$ at $c_{\text {Nip }}=0 \mathrm{~mol} \mathrm{~L}^{-1}$ as shown in Fig. 7a. A comparison of $t_{0, \mathrm{sp}}$ for different metal nanoparticles is done in Table 2 . Also, a linear dependence of $\left(\left(1 / t_{0}\right)-\left(1 / t_{0, \mathrm{sp}}\right)\right) / k$ vs. $\theta_{\mathrm{Nip}}{ }^{2}$
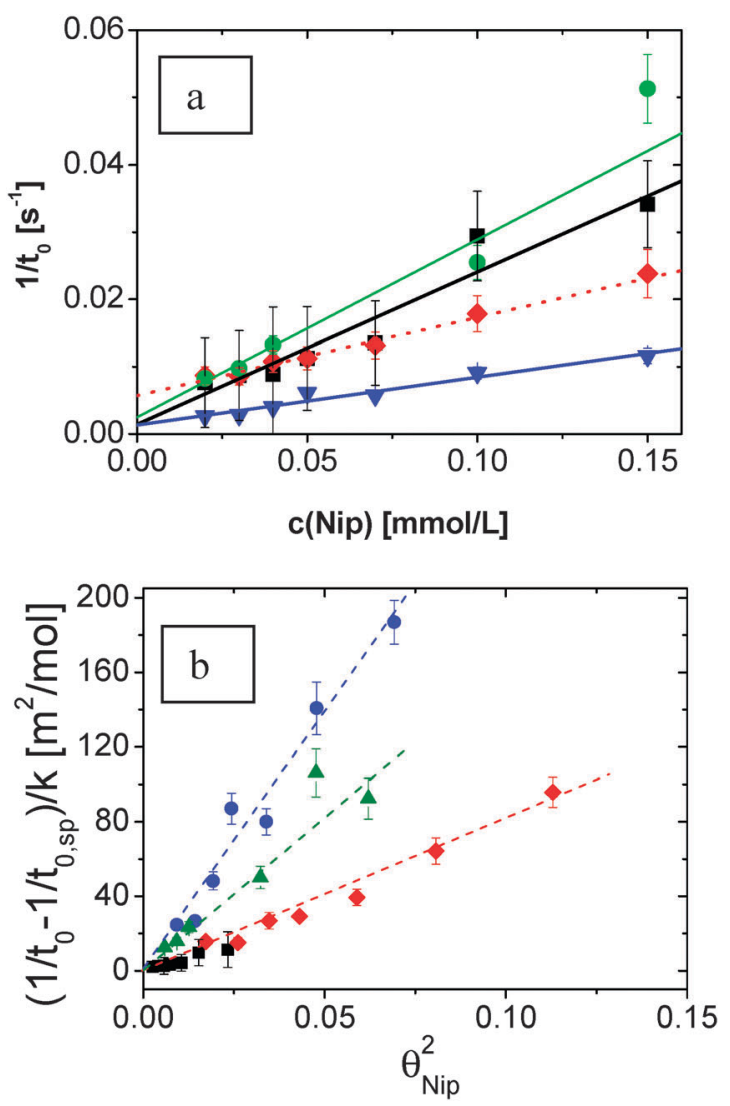

Fig. 7 (a) Dependence of the inverse induction time as a function of the concentration of Nip at a concentration of $10 \mathrm{mM} \mathrm{BH}_{4}$. The different nanoparticles are as follows: black squares: $\mathrm{Au} / \mathrm{Pd}$; blue triangles: Pd; green circles: $\mathrm{Pt}^{13}$; red diamonds: $\mathrm{Au}^{18}$. (b) Dependence of the rate of substrate-induced surface restructuring $\left(\left(1 / t_{0}\right)-\left(1 / t_{0, \mathrm{sp}}\right)\right) / k$ as a function of the surface coverage of 4-nitrophenol $\theta_{\mathrm{Nip}}$ at $10 \mathrm{mM}$ borohydride. The different metal particles are as follows: black squares: $\mathrm{Au} / \mathrm{Pd}$; red diamonds: Au; green triangles: Pt; blue circles: Pd.

(the square of the surface coverage) was found (see Fig. 7b). The values for palladium and gold-palladium particles are compared to platinum ${ }^{13}$ and gold ${ }^{18}$ nanoparticles at $20^{\circ} \mathrm{C}$. This plot is shown in Fig. 7b (red dashed line). For all studied metals and alloys, a linear dependence can be clearly seen in Fig. 7b. The different slope found for different metals indicates the effectivity of the surface-restructuring of the different metals.

Summing up this part we can state that the $\mathrm{Au} / \mathrm{Pd}$ nanoalloys are much better catalysts for the present model reaction than the neat metals. Table 2 demonstrates that this is due to the much enhanced constant $k$ that is higher by one to two orders of magnitude than the data found for neat metals while the adsorption constants are all of similar magnitude. This finding indicates that the adsorbed nitrophenol molecules react with the surface-hydrogen species much faster on the surface of the alloy particles than on the surface of neat metal nanoparticles.

\section{The electronic structure of $\mathrm{Au} / \mathrm{Pd}$ particles}

The experimental data provide strong evidence for a specific ratio of $\mathrm{Au}$ to $\mathrm{Pd}$ yielding optimal catalytic properties of the nanoalloys and underline the importance of surface restructuring. 
These findings suggest that there must be at least two effects that are interplaying to yield the specific $\mathrm{Au} / \mathrm{Pd}$ mixing ratios that are optimal for catalysis: first and obviously the electronic structure must depend on the $\mathrm{Au} / \mathrm{Pd}$ composition, and second, some structural property related to the surface rearrangement must depend on the $\mathrm{Au} / \mathrm{Pd}$ ratio.

However, before adopting this point of view one should critically investigate possible alternatives. A particularly close lying one is that the special catalytic properties could be a pure quantum(size) effect, i.e., a consequence of some special feature in the electronic structure of the alloy particles alone. We have investigated this possibility by calculating the electronic structure of $\mathrm{Au} / \mathrm{Pd}$ clusters from first principles. Classical methods such as molecular dynamics do not give access to electronic properties, thus one has to resort to a quantum mechanical description. This necessarily limits the size of the systems that can be studied: even with the considerable computational power that is available today, the first-principles determination of a metal particle's electronic structure is a serious challenge. A large number of valence electrons per atom and energy surfaces with many minima make calculations for $\mathrm{Au}$ and $\mathrm{Pd}$ particularly challenging.

Our strategy here is to investigate the electronic structure of $\mathrm{Au} / \mathrm{Pd}$ particles as a function of the $\mathrm{Au} / \mathrm{Pd}$ mixing ratio. Nuclear and electronic degrees of freedom are decoupled by the Born-Oppenheimer approximation. The electronic problem is solved in the Kohn-Sham approach of density functional theory with the Perdew-Burke-Ernzerhof Generalized Gradient Approximation $^{27}$ as the exchange-correlation functional. In earlier work we verified that for the observables of noble metals that are of interest here using a Meta-Generalized Gradient Approximation leads to similar results. ${ }^{28}$ We take relativistic effects into account via the effective core potential as implemented in the Turbomole program package. ${ }^{29}$ In particular for $\mathrm{Au}$ we checked for consistency with a normconserving pseudopotential used in a real-space approach. ${ }^{28}$ For extended geometry optimizations the basis set was limited to split valence with one polarization function, but final energies were calculated using a triple zeta basis set with two polarization functions. ${ }^{30} \mathrm{We}$ used the $\mathrm{m} 3$ integration grid in the Turbomole calculations.

Our aim is to check for special electronic properties as a function of $\mathrm{Au} / \mathrm{Pd}$ composition. The effects that we try to identify must be rather general; therefore we study different particle sizes. The smallest particles that we investigate here are 13 atomic clusters, because the structure of $\operatorname{Pd}_{13}$ has recently been established. ${ }^{31}$ Starting from the reported ${ }^{31}$ ground-state geometry of $\mathrm{Pd}_{13}$ we generated alloy clusters by increasing the amount of $\mathrm{Au}$ in a stepwise procedure, replacing $\mathrm{Pd}$ atoms with low coordination numbers by Au atoms. This substitution scheme is suggested by structure optimization studies $^{32,33}$ and is also found in $\mathrm{Au} / \mathrm{Pt}$ particles. ${ }^{28} \mathrm{We}$ then relaxed $^{34}$ the thus constructed starting geometries to minimize the energy. The observable that we here focus on is the electronic density of states (DOS), because a high DOS close to the Fermi edge is typically indicative of electronically active systems. We obtain the DOS of the final, relaxed structure from the Kohn-Sham eigenvalues (see comments below for a justification of this procedure). For plotting the DOS the
Kohn-Sham spectrum has been folded with Gaussians of $0.08 \mathrm{eV}$ width to mimic line broadening. To obtain a quantitative measure we integrated the DOS over an energy interval of $1 \mathrm{eV}$ (from the Fermi edge at $0 \mathrm{eV}$ to $-1 \mathrm{eV}$ ) to define the DOS close to the Fermi edge. The conclusions stated below are independent of the specific value of $1 \mathrm{eV}$, i.e., our conclusions stay the same when the energy integration interval is chosen somewhat larger or smaller. Examples for the DOS and the $1 \mathrm{eV}$ interval are given for larger particles in Fig. 9.

A point that one may expect to request special attention when discussing the DOS is the possible spin polarization (magnetization) of the particles. Bulk $\mathrm{Au}$ and $\mathrm{Pd}$ are not magnetic, but high magnetic moments have been reported for small Pd clusters. ${ }^{31}$ We thus allowed and checked for spin polarization in calculations for the 13 atomic clusters as reported below, evaluating the total energy for different multiplicities. We stress that for the purposes of our study the magnetization is not decisive, as we are interested in the total DOS close to the Fermi level, and we found that this quantity depends very little on the spin polarization in the cases that we studied. The integrated DOS typically changes by less than $1 \%$ for most structures as a function of magnetization, and a few percent at most. For the sake of completeness, however, we also report some magnetizations in the following.

The upper panel of Fig. 8 shows the DOS close to the Fermi edge as a function of the particle composition. The inset depicts the structure of $\mathrm{Au}_{3} \mathrm{Pd}_{10}$ as an example. The total DOS was obtained by summing over both spin channels for that electronic configuration that yields the lowest energy while obeying the Aufbau principle and not showing a degeneracy at the Fermi level. The corresponding numbers of unpaired spins are $6\left(\mathrm{Pd}_{13}\right), 3\left(\mathrm{Pd}_{12} \mathrm{Au}_{1}\right), 4\left(\mathrm{Pd}_{11} \mathrm{Au}_{2}\right)$, and 2 $\left(\mathrm{Pd}_{9} \mathrm{Au}_{4}\right)$. For larger amounts of $\mathrm{Au}$ the plot shows the DOS for configurations with 1 or 0 unpaired spins, depending on whether the electron number is odd or even. From the figure one can clearly draw the conclusion that there are no "particular features" at specific mixing ratios. Instead, there is a clear trend: the DOS at the Fermi edge decreases with increasing $\mathrm{Au}$ amount.

In order to check whether this behaviour of the DOS is general we repeated the procedure for larger clusters. Our choice of further test systems was motivated by the following considerations. Our experimental studies are concerned with relatively large particles that show a bulk-like structure. Therefore, we also theoretically focus on investigating systems where a bulk-like geometry is a reasonable starting point. Furthermore, as bulk $\mathrm{Pd}$ and $\mathrm{Au}$ are not magnetic, and as our experimental particles are bulk-like in their structure, it is a plausible assumption that magnetic effects are not important for explaining the catalytic properties. In addition, our study of the 13 atomic clusters has shown that the total DOS is not very sensitive to the magnetization. Therefore, we choose for our following investigations always the electronic configuration that has the lowest spin polarization (under the condition that the Aufbau principle is respected).

From the just described points of view the 20 atomic clusters are good candidates for the theoretical investigation due to the established fcc-like structure ${ }^{35}$ of $\mathrm{Au}_{20}$. In this tetrahedral geometry we replaced $\mathrm{Au}$ by $\mathrm{Pd}$ in a step-by-step manner, 

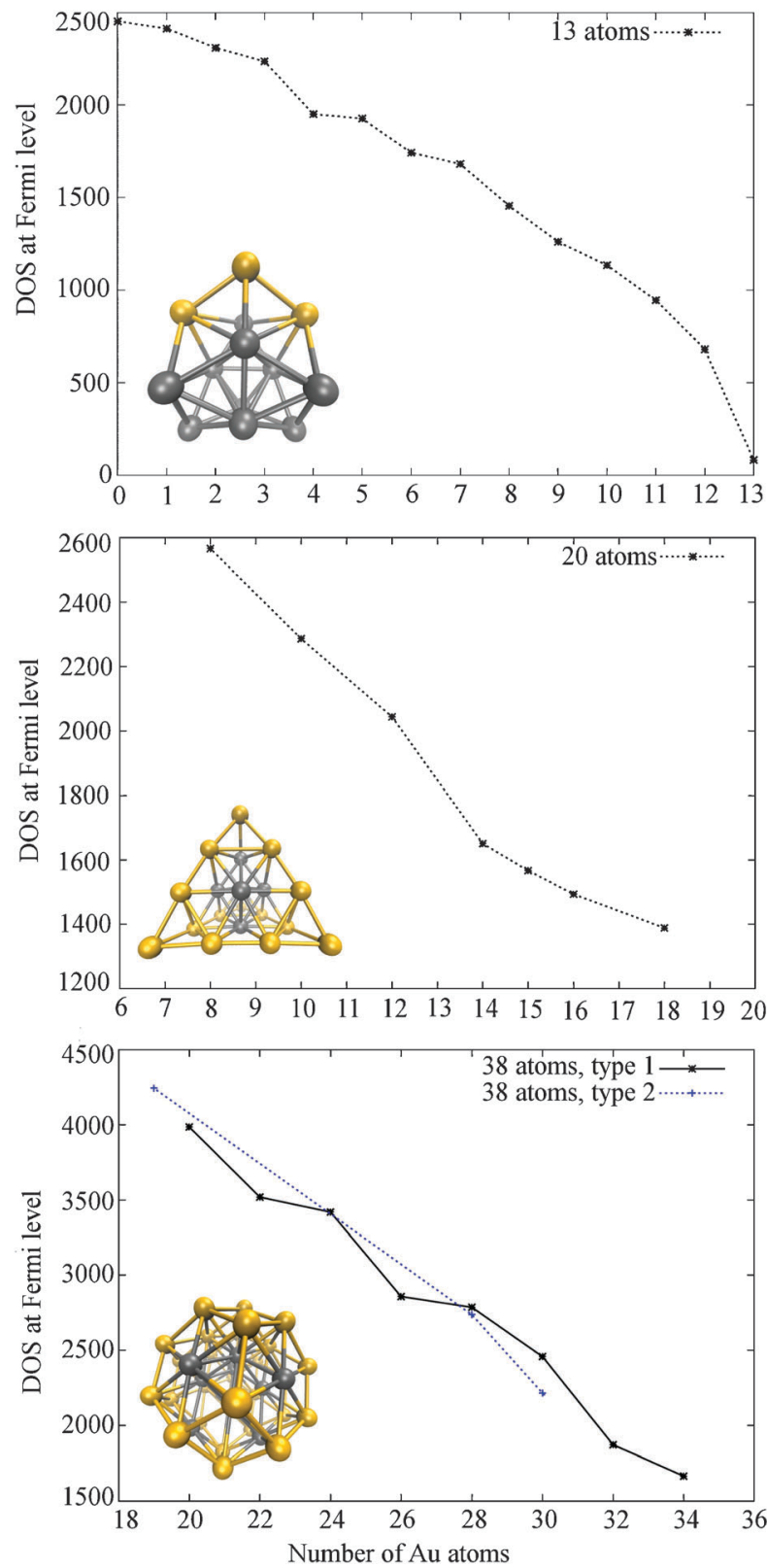

Fig. 8 The density of states close to the Fermi level as a function of the $\mathrm{Au}$ content for $\mathrm{Au} / \mathrm{Pd}$ particles. For all three sizes (from top to bottom: 13 atomic, 20 atomic and 38 atomic) the density of states decreases with increasing Au content. For the 38 atomic clusters the density of states for two different structure types is shown. The trend is the same for both types.

again observing the rule that central (high-coordination number) sites are energetically favourable for $\mathrm{Pd}$. The middle panel of Fig. 8 shows the structure of $\mathrm{Au}_{16} \mathrm{Pd}_{4}$ as an example and the DOS at the Fermi edge as a function of the Au content. Also in this case a decrease with increasing amount of $\mathrm{Au}$ is observed for all mixed $\mathrm{Au} / \mathrm{Pd}$ particles. This observation does not depend on the specific structural motif. To demonstrate this explicitly and to check another cluster size we repeated the procedure for systems with 38 atoms. This number was chosen because it allows for building a truncated octahedron, thus mimicking the bulk-like faceted structure of the larger particles that are observed in experiment. We studied two lines of structures with 38 atoms. In both of them $6 \mathrm{Pd}$ atoms form the core, as this is an energetically favoured arrangement. However, the two lines differ in the position of the remaining "surface" Pd atoms. The structures labelled "type 1" in the lower panel of Fig. 8 were obtained by randomly replacing surface Au by Pd atoms. In the structures labelled "type 2" the surface $\mathrm{Pd}$ atoms were placed such that they occupy the interior parts of the facets. In the literature ${ }^{32}$ these latter configurations have been reported as being energetically favourable. Our calculations confirm this finding as structures of type 2 are lower by $0.08 \mathrm{eV}$ for $\mathrm{Au}_{30} \mathrm{Pd}_{8}$ and $0.83 \mathrm{eV}$ for $\mathrm{Au}_{28} \mathrm{Pd}_{10}$ than the corresponding structures of type 1 . We observe that all structures have rather small HOMO-LUMO gaps, which may be an indication for further symmetry breaking being energetically favourable. However, as explained above, we here deliberately focus on the bulk-like structures as we use them as small-scale models for the larger, experimentally observed structures that are faceted and fcc-like.

The important finding is that also in the 38 atomic faceted cases both types of structures show the same trend: the DOS at the Fermi edge falls with increasing $\mathrm{Au}$ content without a composition specific maximum. Thus, our results confirm that there is no specific feature in the DOS alone that could explain the enhanced catalytic activity.

This finding is in line with the experimental indications for the importance of effects that involve an interplay of structural and electronic properties. Therefore, we investigate in the following the relation between the clusters' geometry and their electronic structure. To this end we take a closer look at several of the faceted 38 atomic particles. Fig. 9 shows from top to bottom $\mathrm{Au}_{32} \mathrm{Pd}_{6}, \mathrm{Au}_{30} \mathrm{Pd}_{8}$, three different geometries for $\mathrm{Au}_{28} \mathrm{Pd}_{10}$, and $\mathrm{Au}_{19} \mathrm{Pd}_{19}$. The right column shows the occupied DOS with the highest occupied eigenvalues aligned at $0 \mathrm{eV}$. The vertical line at $-1 \mathrm{eV}$ indicates the previously mentioned integration range. The left column shows the atomic geometry and a visualization of the highest-occupied molecular orbital (HOMO). The HOMO is particularly relevant as it represents the energetically high-lying part of the electronic density that is most reactive, typically has the largest spatial extent, and is thus of special importance for binding effects. We also stress that contrary to earlier beliefs the orbitals from Kohn-Sham Density Functional Theory bear physical significance. This has not only been demonstrated very early for metal clusters, ${ }^{36}$ but also has been generally rationalized on the grounds of Kohn-Sham theory. ${ }^{37-39}$ Not only the Kohn-Sham energy eigenvalues but even orbital shapes are physically meaningful. ${ }^{40,41}$

The first observation in Fig. 9 is that the HOMO in each case is closely associated with the $\mathrm{Pd}$ atoms. The second observation is that an increasing amount of $\mathrm{Pd}$, which brings an increasing number of Pd atoms to the surface, thus leads to a HOMO that is dominantly located at the surface. This is clearly seen, e.g., when comparing the HOMO representation of $\mathrm{Au}_{32} \mathrm{Pd}_{6}$ to the one of $\mathrm{Au}_{19} \mathrm{Pd}_{19}$. This can indicate that $\mathrm{Pd}$ atoms at the surface are likely to be favourable in terms of the particles' activity.

Third, we carefully inspect three different isomers of $\mathrm{Au}_{28} \mathrm{Pd}_{10}$. Structure (e) as suggested in the literature ${ }^{32}$ has 


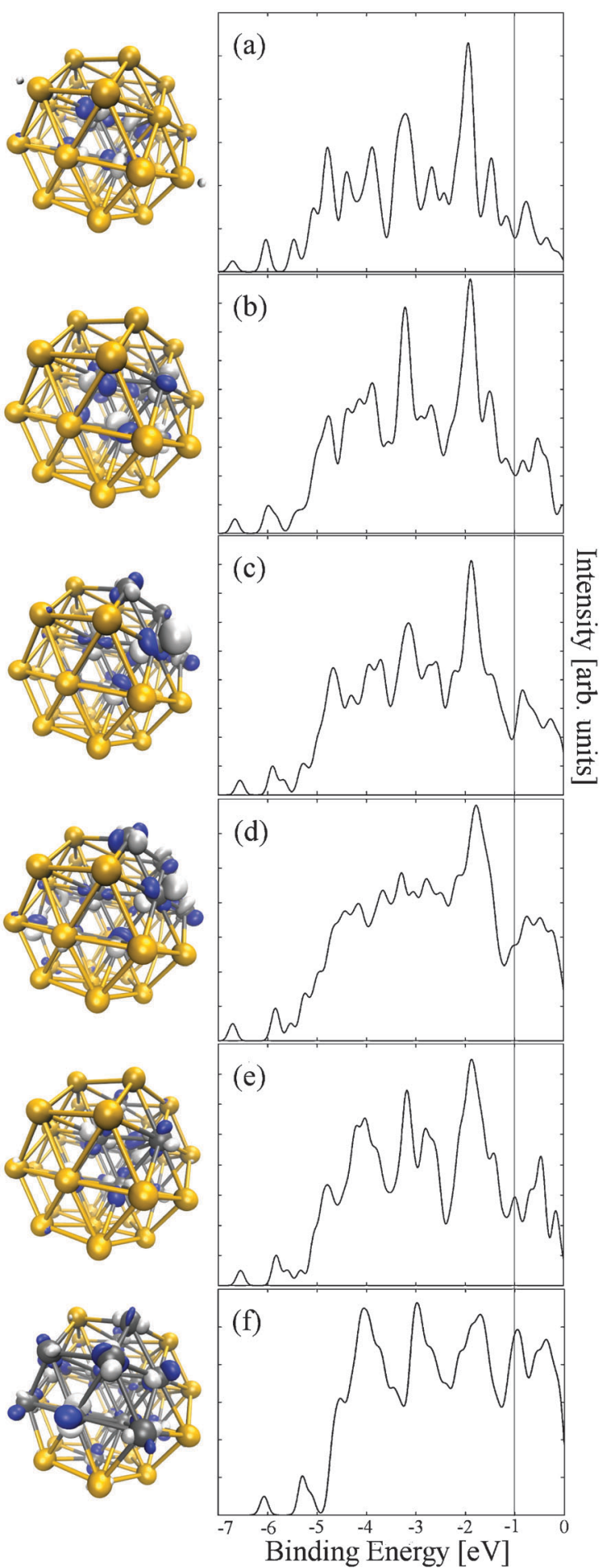

Fig. 9 Left: geometric structure and isosurface plot of the HOMO of 38 atomic particles. From top to bottom: (a): $\mathrm{Au}_{32} \mathrm{Pd}_{6}$, (b): $\mathrm{Au}_{30} \mathrm{Pd}_{8}$, (c)-(e): 3 different structures for $\mathrm{Au}_{28} \mathrm{Pd}_{10}$, (f): $\mathrm{Au}_{19} \mathrm{Pd}_{19}$. White and blue areas represent negative and positive isosurface values of 0.04 atomic units. Right: the density of states (DOS) for each structure. The HOMO is associated with the Pd atoms, and an increasing amount of Pd leads to an increased HOMO density at the particles' surface.

the lowest total energy, (c) is higher by $0.83 \mathrm{eV}$ and (d) by $1.64 \mathrm{eV}$. We do not believe that the energetic ordering here is particularly relevant for the relation to the experiment, because the conditions in the experiment are very complex. The experimental structures may be determined by the synthesis procedure, the presence of the polyelectrolyte brushes, and the solvent. However, independent of these differences, there is an important observation to be made in comparing structures (c)-(e): although the three geometries are overall rather similar, the differences between the HOMOs are quite noticeable. A moderate change in the geometry leads to a significant change of the energetically high-lying part of the density.

Thus, a specific arrangement of $\mathrm{Au}$ and $\mathrm{Pd}$ atoms at the surface can lead to special electronic and thus chemical properties. This effect is larger for binary clusters than for pure ones because of the additional variations that arise from the different atomic species. Thus, the surface structure, which is well known to be important in catalysis and nanoparticles in general, is particularly complex and important in nanoalloys. These findings are in line with the conclusions drawn from the experiments.

\section{Conclusions}

In conclusion, colloidal stable and homogeneous gold-palladium nanoalloy particles can be prepared and immobilized on cationic spherical polyelectrolyte brushes (SPBs). HR-TEM, FFT and PXRD measurements demonstrate that well-defined faceted $\mathrm{Au} / \mathrm{Pd}$ nanoalloys are generated in the system. The catalytic activity of the $\mathrm{Au} / \mathrm{Pd} @ \mathrm{SPB}$ nanoalloys has been tested by reduction of 4-nitrophenol. The rate constant normalized to the metal surface area goes through a maximum as a function of the $\mathrm{Au} / \mathrm{Pd}$ composition. The kinetic analysis demonstrates that this enhancement is due to a much higher reactivity of the reacting species on the surface. DFT calculations show that the density of states close to the Fermi edge is dominantly associated with the $\mathrm{Pd}$ atoms and that small structural rearrangements can have a considerable impact on the particles' surface properties. The sum of experimental and theoretical findings points to an enhanced reactivity of the surface in nanoalloys.

\section{Acknowledgements}

We acknowledge financial support by the German Science Foundation, SFB 840, Bayreuth.

\section{Notes and references}

1 R. Narayanan, Molecules, 2010, 15, 2124-2138.

2 J. M. Campelo, D. Luna, R. Luque, J. M. Marinas and A. A. Romero, ChemSusChem, 2009, 2, 18-45.

3 A. Haruta, Chem. Rec., 2003, 3, 75-87.

4 R. Ferrando, J. Jellinek and R. L. Johnston, Chem. Rev., 2008, 108, 845-910.

5 V. V. G. N. R. Shiju, Appl. Catal., A, 2009, 356, 1-17.

6 G. J. Hutchings, Dalton Trans., 2008, 5523-5536.

7 M. Schrinner, S. Proch, Y. Mei, R. Kempe, N. Miyajima and M. Ballauff, Adv. Mater., 2008, 20, 1928-1933.

8 M. Schrinner, M. Ballauff, Y. Talmon, Y. Kauffmann, J. Thun, M. Moller and J. Breu, Science, 2009, 323, 617-620.

9 M. Ballauff, Prog. Polym. Sci., 2007, 32, 1135-1151.

10 Y. Lu, A. Wittemann and M. Ballauff, Macromol. Rapid Commun., 2009, 30, 806-815.

11 Y. Mei, Y. Lu, F. Polzer, M. Ballauff and M. Drechsler, Chem. Mater., 2007, 19, 1062-1069. 
12 Y. Mei, G. Sharma, Y. Lu, M. Ballauff, M. Drechsler, T. Irrgang and R. Kempe, Langmuir, 2005, 21, 12229-12234.

13 S. Wunder, F. Polzer, Y. Lu, Y. Mei and M. Ballauff, J. Phys. Chem. C, 2010, 114, 8814-8820.

14 Y. Lu, Y. Mei, R. Walker, M. Ballauff and M. Drechsler, Polymer, 2006, 47, 4985-4995.

15 N. Pradhan, A. Pal and T. Pal, Colloid Surf., A, 2002, 196, $247-257$.

16 S. K. Ghosh, M. Mandal, S. Kundu, S. Nath and T. Pal, Appl. Catal., A, 2004, 268, 61-66.

17 K. Esumi, K. Miyamoto and T. Yoshimura, J. Colloid. Interface Sci., 2002, 254, 402-405.

18 S. Wunder, Y. Lu, M. Albrecht and M. Ballauff, ACS Catal., 2011, 1, 908-916.

19 L. Y. Chang, A. S. Barnard, L. C. Gontard and R. E. DuninBorkowski, Nano Lett., 2010, 10, 3073-3076.

20 X. Guo, A. Weiss and M. Ballauff, Macromolecules, 1999, 32, 6043-6046.

21 M. R. Knecht, M. G. Weir, A. I. Frenkel and R. M. Crooks, Chem. Mater., 2008, 20, 1019-1028.

22 M. Simoes, S. Baranton and C. Coutanceau, J. Phys. Chem. C, 2009, 113, 13369-13376.

23 Y. Khalavka, J. Becker and C. Sonnichsen, J. Am. Chem. Soc., 2009, 131, 1871-1875.

24 S. Panigrahi, S. Basu, S. Praharaj, S. Pande, S. Jana, A. Pal, S. K. Ghosh and T. Pal, J. Phys. Chem. C, 2007, 111, 4596-4605.

25 J. Zeng, Q. Zhang, J. Y. Chen and Y. N. Xia, Nano Lett., 2010, 10, 30-35.

26 G. Ertl, Faraday Discuss., 2002, 121, 1-15.

27 J. P. Perdew, K. Burke and M. Ernzerhof, Phys. Rev. Lett., 1996, 77, 3865-3868.

28 L. Leppert and S. Kümmel, J. Phys. Chem. C, 2011, 115, 6694-6702.
29 R. Ahlrichs, M. Bär, M. Häser, H. Horn and C. Kölmel, Chem. Phys. Lett., 1989, 162, 165-169.

30 F. Weigend and R. Ahlrichs, Phys. Chem. Chem. Phys., 2005, 7, 3297.

31 A. M. Koster, P. Calaminici, E. Orgaz, D. R. Roy, J. U. Reveles and S. N. Khanna, J. Am. Chem. Soc., 2011, 133, 12192-12196.

32 F. Pittaway, L. O. Paz-Borbon, R. L. Johnston, H. Arslan, R. Ferrando, C. Mottet, G. Barcaro and A. Fortunelli, J. Phys. Chem. C, 2009, 113, 9141-9152.

33 I. V. Yudanov and K. M. Neyman, Phys. Chem. Chem. Phys., 2010, 12, 5094-5100.

34 According to ref. 31 geometries for different spin states are very similar, therefore the relaxation was done for one spin configuration only in each case and then the spin polarization was varied at fixed geometry to determine the lowest energy spin state.

35 J. Li, X. Li, H. J. Zhai and L. S. Wang, Science, 2003, 299, 864.

36 J. Akola, M. Manninen, H. Häkkinen, U. Landman, X. Li and L. S. Wang, Phys. Rev. B: Condens. Matter, 2000, 62, 13216-13228.

37 D. P. Chong, O. V. Gritsenko and E. J. Baerends, J. Chem. Phys., 2002, 116, 1760-1772.

38 T. Körzdörfer, S. Kümmel, N. Marom and L. Kronik, Phys. Rev. B: Condens. Matter, 2009, 79, 201205; T. Körzdörfer, S. Kümmel, N. Marom and L. Kronik, Phys. Rev. B: Condens. Matter, 2010, 82, 129903(E).

39 T. Körzdörfer and S. Kümmel, Phys. Rev. B: Condens. Matter, 2010, 82, 155206.

40 P. Puschnig, S. Berkebile, A. J. Fleming, G. Koller, K. Emtsev, T. Seyller, J. D. Riley, C. Ambrosch-Draxl, F. P. Netzer and M. G. Ramsey, Science, 2009, 326, 702-706.

41 M. Dauth, T. Körzdörfer, S. Kümmel, J. Ziroff, J. Wiessner, A. Schöll, F. Reinert, M. Arita and K. Shimada, Phys. Rev. Lett., 2011, 107, 193002-193005. 